\title{
Déterminisme génétique de la tolérance de Lagenaria siceraria (Molina) Standley (Cucurbitaceae) aux insectes ravageurs et recherche de marqueurs microsatellites SSR associés aux gènes
}

\section{Anzara Guy Roland}

UFR Agroforesterie, Université Jean Lorougnon Guède, Daloa, Côte d'Ivoire Angui Chia Michelle Valérie

Unité de Formation et de Recherche des Sciences de la nature, Université Nangui Abrogoua, Côte d'Ivoire

\section{Doubi Bi Tra Serges}

Centre National de Recherche Agronomique, Station de recherche Marc Delorme, Côte d'Ivoire

\section{Akaffou Doffou Selastique}

UFR Agroforesterie, Université Jean Lorougnon Guède, Daloa, Côte d'Ivoire Zoro Bi Irié Arséne

Unité de Formation et de Recherche des Sciences de la nature, Université Nangui Abrogoua, Côte d'Ivoire

\section{Doi:10.19044/esj.2021.v17n10p233}

Submitted: 30 January 2021

Accepted: 08 February 2021

Published: 31 March 2021
Copyright 2021 Author(s)

Under Creative Commons BY-NC-ND

4.0 OPEN ACCESS

Cite As:

Roland A.G., Michelle Valérie A.C., Serge D.B.T., Selastique A.D. \& Arséne Z.B.I. (2021). Déterminisme génétique de la tolérance de Lagenaria siceraria (Molina) Standley (Cucurbitaceae) aux insectes ravageurs et recherche de marqueurs microsatellites SSR associés aux gènes. European Scientific Journal, ESJ, 17(10), 233.

https://doi.org/10.19044/esj.2021.v17n10p233

\section{Résumé}

La gourde oléagineuse, Lagenaria siceraria (Molina) Standley, se caractérise par un haut potentiel nutritionnel et une forte valeur marchande dont l'intensification de la production pourrait profiter aux agriculteurs, en particulier, les femmes rurales qui en sont les principales productrices. Malgré l'importance socio-économique de la gourde oléagineuse, la production est caractérisée par de faibles rendements, dus essentiellement aux dégâts occasionnés par les insectes ravageurs. La présente étude vise à connaitre le 
déterminisme génétique de la tolérance de Lagenaria siceraria aux insectes ravageurs et à identifier les marqueurs moléculaires proches des QTLs contrôlant cette tolérance. Cette identification serait une étape importante pour accélérer le processus de la tolérance de L. siceraria aux insectes ravageurs. Des marqueurs moléculaires de type SSR ont été évalués sur 100 individus de la population F2 (issue de l'autofécondation de la F1 provenant du croisement entre les accessions parentales NI431 et NI227). Un seul QTL dénommé qTDco a été identifié et le marqueur C1.2.23 a été associé à ce QTL. Ce marqueur, utilisé, dans un programme de sélection assistée par marqueur, serait un atout considérable dans le processus de la recherche de la tolérance de $L$. siceraria aux insectes ravageurs.

Mots-clés: Lagenaria siceraria, insectes ravageurs, population en ségrégation F2, marqueurs SSR, QTL de la tolérance

\title{
Identification for SSR microsatellite markers associated with genes conferring tolerance of Lagenaria siceraria (Molina) Standley (Cucurbitaceae) to insect pests
}

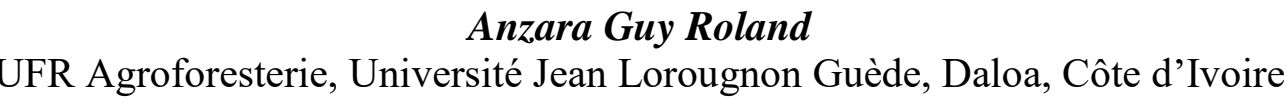
Angui Chia Michelle Valérie

Unité de Formation et de Recherche des Sciences de la nature,

Université Nangui Abrogoua, Côte d'Ivoire

\section{Doubi Bi Tra Serges}

Centre National de Recherche Agronomique,

Station de recherche Marc Delorme, Côte d'Ivoire

Akaffou Doffou Selastique

UFR Agroforesterie, Université Jean Lorougnon Guède, Daloa, Côte d'Ivoire

\section{Zoro Bi Irié Arséne}

Unité de Formation et de Recherche des Sciences de la nature,

Université Nangui Abrogoua, Côte d'Ivoire

\begin{abstract}
Lagenaria siceraria (Molina) Standley is characterized by high nutritional potential and high market value. The intensification of $L$. siceraria could benefit farmers, especially rural women who are the main farmers. Despite the socio-economic importance of this oil gourd, its production is characterized by low yields, mainly due to damage caused by insect pests. The present study aims to identify molecular markers SSR associated to QTLs
\end{abstract}


controlling the tolerance of insect damage. Thoses molecular markers would be an important step in accelerating the process of tolerance of $L$. siceraria to insect pests. Molecular markers microsatellites SSR were evaluated on 100 individuals of the $\mathrm{F} 2$ population (resulting from the self-fertilization of $\mathrm{F} 1$ from the crossing between parental accessions NI431 and NI227). A single QTL called qTDco was identified and the marker C1.2.23 was associated with this QTL. This marker used in a marker-assisted selection program would be a considerable asset in the process of researching the tolerance of $L$. siceraria to insect pests.

Keywords: Lagenaria siceraria; insect pests, F2 segregated population; SSR markers, tolerance QTL

\section{Introduction}

L'économie de nombreux pays, en Afrique, repose, essentiellement, sur l'agriculture. La forte démographie, observée, au cours des dernières années et la forte pression parasitaire sur les cultures, ont contribué à une baisse de la production agricole. De nombreuses initiatives ont, donc, été entreprises pour améliorer le rendement. Une politique d'introduction de nouvelles variétés, à haut rendement, a, ainsi, été développée (Akpavi et al., 2013). Celles-ci, bien adoptées par les paysans ont permis de faire reculer la faim et surtout d'améliorer leurs revenus. Cette initiative a toutefois entrainé le délaissement des variétés traditionnelles, source de diversité génétique (Baudoin, 2001 ; Rasul et al., 2007). Cet abandon est plus accentué pour certaines plantes d'utilisation locale et négligées par les programmes de recherches et de développement. C'est le cas des cucurbitacées oléagineuses communément appelées "pistache" en Côte d'Ivoire (Ndabalishye, 1995) et "Egussi" au Nigeria et Bénin (Achigan et al., 2006). Parmi elles, figure Lagenaria siceraria (Molina) Standley (Cucurbitaceae) qui est largement utilisée au cours des prestigieuses fêtes populaires et cérémonies traditionnelles (Zoro Bi et al., 2006). Aussi, elle représente un atout considérable pour l'accroissement du revenu des paysans d'autant plus que la valeur marchande des graines avec un prix moyen de 2 à $3 € / \mathrm{kg}$ (environ 1500 à $2000 \mathrm{~F} \mathrm{CFA}$ ) est parfois bien plus élevée que celle des cultures dites d'exportation (Zoro Bi et al., 2003). De plus, elles sont très riches en lipides et en protéines (Loukou et al., 2007).

Malgré son importance économique et nutritionnelle, le rendement de $L$. siceraria demeure très faible dans les zones de production (Zoro Bi et al., 2003). Cette faible production de L. siceraria serait due à plusieurs bioagresseurs, particulièrement les insectes (Vayssieres et al., 2000 ; Pitan et Ekoja, 2011). Le prix très élevé des insecticides chimiques et l'impact négatif de leur utilisation sur l'environnement limite fortement leur utilisation par les producteurs ruraux. L'introduction des plantes résistantes dans le système de 
production devient ainsi une solution appropriée (Najimi et al., 2003). Toutefois, l'utilisation des variétés résistantes ou tolérantes aux insectes peut être de courtes durées car certains gènes de la résistance/tolérance pourraient être rapidement contournés par le développement de nouveaux biotypes. Il faut encore un temps relativement long pour rechercher de nouvelles sources de variétés résistantes (Rouxel et al., 2003).

L'une des solutions pour résoudre le problème lié à l'adaptation de nouveaux agents pathogènes est l'identification des marqueurs moléculaires proches des QTLs impliqués dans la résistance/tolérance aux insectes ravageurs. Cette identification serait une étape importante pour accélérer le processus de la tolérance de $L$. siceraria aux insectes ravageurs. En effet, des plantes porteuses de gènes de la tolérance peuvent être sélectionnées même à un stade précoce en se basant uniquement sur la présence de certains marqueurs chez ces plantes.

C'est dans ce contexte que se situe la présente étude, qui vise à examiner le nombre de gènes et à identifier les marqueurs moléculaires, de type SSR (Single Sequence Repeat) proches des QTL (Quantitaive Trait Loci) de tolérance de L. siceraria aux insectes ravageurs à partir de la population en ségrégation F2 issu du croisement entre une accession tolérante (NI431) et une accession sensible (NI227) aux dégâts foliaires des insectes.

\section{Matériel et Méthode Zone d'étude}

Les expérimentations ont été conduites en 2010 dans le village de Manfla (6 $49^{\prime} 34.38^{\prime} ' \mathrm{~N}, 5^{\circ} 43^{\prime} 47.68^{\prime}$ ' $\left.\mathrm{W}\right)$, zone de forte production des cucurbites, situé à $400 \mathrm{Km}$, au nord d'Abidjan (Côte d'Ivoire). La zone est caractérisée par deux saisons pluvieuses séparées par une courte période sèche (Juillet-Août) et une longue saison sèche (Décembre-Février). La pluviométrie et la température moyenne annuelle sont, respectivement, de $1200 \mathrm{~mm}$ et de $27^{\circ} \mathrm{C}$. La végétation est de type savane arborée. les caractéristiques du sol, à $20 \mathrm{~cm}$ de profondeur, sont les suivantes: $\mathrm{pH}=6,45$, avec $57 \%$ de sable, $36 \%$ de limon, $7 \%$ d'argile, $6 \%$ de matière organique, $3,5 \mathrm{~g} / \mathrm{Kg}$ de $\mathrm{N}$ total, $24,4 \mathrm{~g} / \mathrm{Kg}$ de $\mathrm{P}$ disponible et $0,45 \mathrm{~g} / \mathrm{Kg}$ de $\mathrm{K}$ (Kouassi et Zoro Bi, 2009).

\section{Matériel végétal}

Les différents génotypes utilisés dans cette étude ont été les populations parentales, NI431 et NI227 constituées de 25 individus chacune. Les accessions NI431 et NI227 ont été considérées, respectivement, comme, tolérantes et sensibles aux attaques des insectes ravageurs (Anzara et al., 2015). Le croisement entre ces deux populations, à travers la pollinisation manuelle a été effectué dans les deux sens. Ceci a permis d'obtenir 50 individus F1 (Figure 1). Une autofécondation des individus de la F1 a donné 
(dont deux fruits par individus) la population en ségrégation F2 composée de 100 individus.
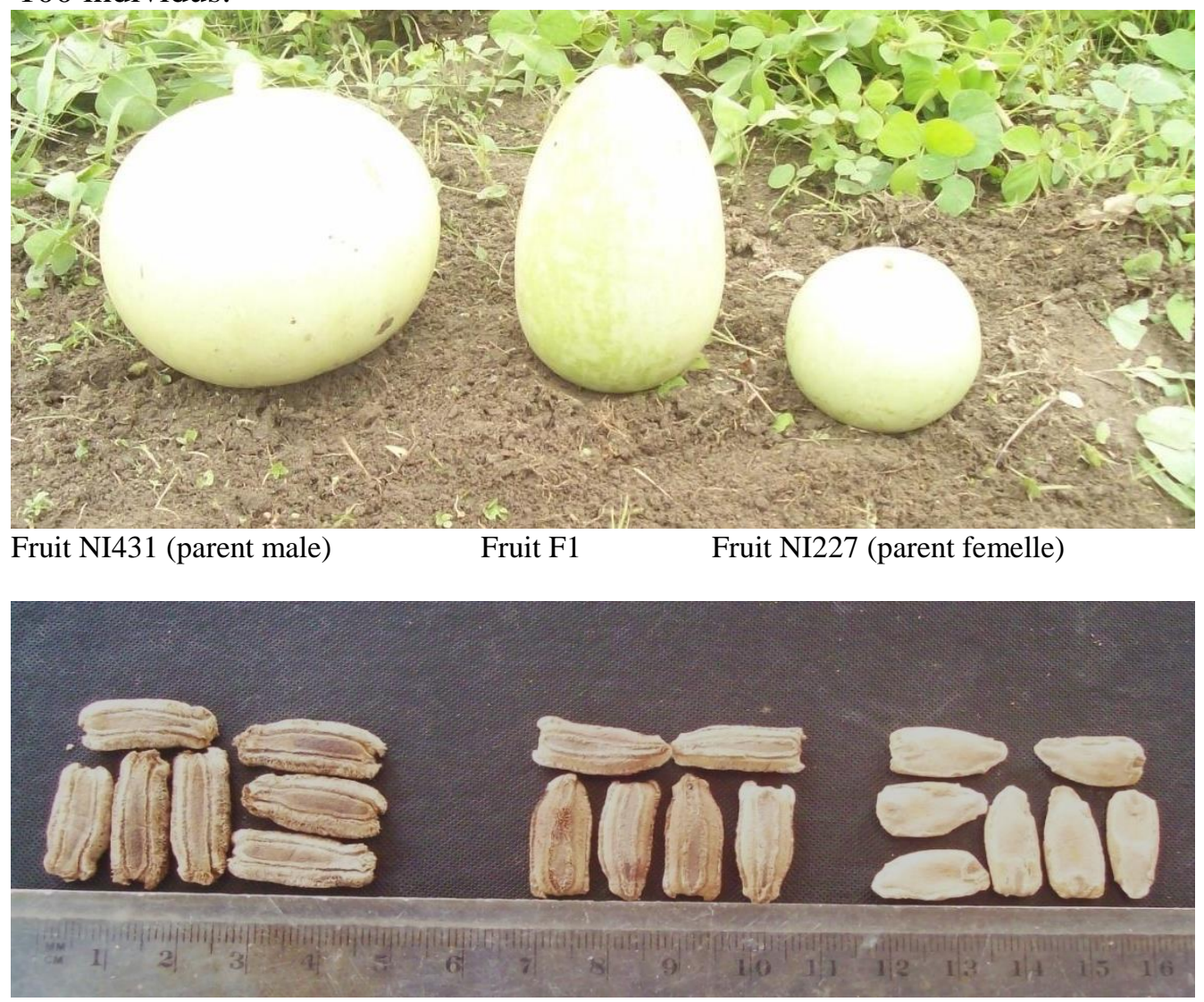

Graine NI431 (parent male)

Graine F1

Graine NI227 (parent femelle)

Figure 1. Populations parentales et en ségrégation utilisées

\section{Dispositif expérimental}

Cent cinquante (150) individus dont 100 pour la population F2 et 25 pour chaque population parentale ont été utilisés. Les graines des 150 individus ont été semées sur une parcelle de $1500 \mathrm{~m}^{2}(75 \mathrm{~m} \times 20 \mathrm{~m})$. La distance entre deux points de semis, sur la même ligne, était de $3 \mathrm{~m}$ et la distance entre les lignes a été également de $3 \mathrm{~m}$. l'évaluation des dégâts foliaires a été effectuée selon Anzara et al. 2015 à partir de deux paramètres : le Pourcentage de Feuilles attaquées (PoFa) et la Sévérité des Dégâts (SeDe).

Le Pourcentage de feuilles attaquées a été calculé en utilisant la formule suivante :

$$
P o F a=\frac{n F a}{n F t} \times 100
$$


$\mathrm{nFa}$ : nombre de feuilles détruites par les insectes; $\mathrm{nFt}$ : nombre de feuilles totales d'une plante

La sévérité des dégâts a été évaluée sur la surface foliaire détruite de l'ensemble des feuilles attaquées par les insectes. Elle a été évaluée en attribuant une note à chaque plante. La note a varié de 0 à 5 en fonction de la proportion des dégâts (Bubici \& Cirulli, 2008 ; Sobrinho et al., 2010). Ainsi, 0 correspond-il à l'absence totale des dégâts observés sur les feuilles et la note 5 indiquant la mort de la plante. La note 1 a concerné les plantes dont les dégâts foliaires se situaient entre 1 à $25 \%$. Les plantes dont 26 à $50 \%$ de la surface foliaire était détruite ont eu la note 2 . Les notes 3 et 4 ont été attribuées, respectivement aux plantes dont 51 à $75 \%$ et 76 à $100 \%$ de surfaces foliaires étaient détruites.

Les feuilles de chaque individu ont été prélevées et séchées pour l'extraction de l'ADN.

\section{Extraction d'ADN}

Le matériel végétal qui a servi aux analyses moléculaires était composé d'une jeune feuille séchée prise sur chacun des 25 individus de chacune des deux populations parentales (NI431 et NI227) et sur chacun des 100 individus de la population en ségrégation F2.

L'ADN génomique des différents individus a été extrait à partir des fragments de 50 à $70 \mathrm{mg}$ de jeunes feuilles sèches suivant la méthode modifiée de Murray et Thompson (1980). Le broyage des feuilles a été effectué dans l'azote liquide dans des tubes Eppendorfs. La poudre de feuille sèche obtenue a été reprise dans $700 \mu \mathrm{L}$ de tampon d'extraction CTAB (Cetyl Trimethyl Ammonium Bromide). Cette solution contenait 2\% de CTAB, 1,4 M de $\mathrm{NaCl}$, 0,1 $\mathrm{M}$ de Tris-base, $20 \mathrm{mM}$ d'acide éthylène-diamine-tétraacétique (EDTA) et $2 \%$ de polyvinylpyrrolidone (PVP-40). L'ensemble broyat de feuille sèche plus tampon d'extraction contenu dans un tube Eppendorf, a été incubé au bain-marie à $65^{\circ} \mathrm{C}$ pendant 30 minutes. Toutes les 10 minutes le mélange contenu dans chacun des tubes Eppendorf a été homogénéisé par agitation. Au terme des 30 minutes d'incubation, les tubes Eppendorfs ont été retirés du bain-marie, refroidis à température ambiante pendant deux minutes puis centrifugés à $19000 \mathrm{~g}$ pendant 10 minutes. Une quantité de $500 \mu \mathrm{L}$ du surnageant a été prélevée puis complétée avec $500 \mu \mathrm{L}$ de Chloroforme. Ce mélange a été centrifugé pendant 5 min à $19000 \mathrm{~g}$. La phase aqueuse $(300 \mu \mathrm{L})$ a été transvasée dans un nouveau tube Eppendorf puis complétée avec $350 \mu \mathrm{L}$ de chloroforme. Ce mélange a été encore centrifugé dans les mêmes conditions que précédemment. L'ADN a été ensuite précipité pendant une heure à $-20^{\circ} \mathrm{C}$ avec $350 \mu \mathrm{L}$ d'isopropanol. Après une centrifugation (16000 g pendant 10 minutes à température ambiante), le culot a été lavé avec $500 \mu \mathrm{L}$ d'éthanol $70 \%$. Cette opération a été répétée puis l'ADN a été séché à $37^{\circ} \mathrm{C}$ pendant 30 
min. L'ADN a été conservé dans $100 \mathrm{ml}$ de tampon d'élution TE composé de $10 \mathrm{mmol} / \mathrm{L}$ de Tris- $\mathrm{HCl}$ et $1 \mathrm{mmol} / \mathrm{L}$ de EDTA à $\mathrm{pH}$ 8.0.Une migration de contrôle sur gel d'agarose $1 \%$ des extraits brutes d'ADN a été effectuée pour tester leurs qualités. L'électrophorèse sur gel d'agarose à $1 \%$ a été soumise à $60 \mathrm{~mA}$ pendant $20 \mathrm{~min}$. Pour cette migration, le tampon d'électrophorèse TrisBorate EDTA (TBE) $1 \mathrm{mM} \mathrm{pH} 8$ a été utilisé. La concentration en ADN des différentes accessions a été déterminée en comparant l'intensité des bandes obtenues après migration sur gel d'agarose à celle de l'ADN standard Smart Ladder.

\section{Amplification de l'ADN microsatellite par la méthode PCR}

Six amorces SSR ont été utilisées dans cette étude. Ces amorces se sont révélées polymorphes parmi celles utilisées au cours du développement des marqueurs microsatellites portant sur l'étude de la diversité génétique de la gourde oléagineuse. La PCR a été réalisée en présence de deux amorces, l'amorce reverse $(\mathrm{R})$ et l'amorce forward $(\mathrm{F})$ contenant une queue M13 marquée à un fluorophore absorbant à des longueurs d'onde de 700 ou 800 nanomètres. Les noms de ces marqueurs, leurs séquences, les motifs de base et leur température d'hybridation sont présentés dans le tableau 1.

Tableau 1. Liste des amorces utilisées, leurs séquences et leur température d'hybridation.

\begin{tabular}{|c|c|c|c|}
\hline Noms & Motif & Séquences & $\operatorname{Tm}\left({ }^{\circ} \mathbf{C}\right)$ \\
\hline URF1 & $(\mathrm{CTAG}) 3$ & $\begin{array}{l}\text { F: AGCAGCACCTTGTCTTGTAT } \\
\text { R: CACAGATCCCACTCAATCTT }\end{array}$ & 55 \\
\hline EST00691 & (CTC)4 & $\begin{array}{l}\text { F: CCAAACCCTTCTCTTTCTTT } \\
\text { R: AAGGAGGTGAGTGAAGGTG }\end{array}$ & 55 \\
\hline EST00680 & (TG)5(TTCC)5 & $\begin{array}{l}\text { F: CCTTATCTCAACTCTTTTCGGA } \\
\text { R: AGGATTGGGCTTGATTGTTG }\end{array}$ & 55 \\
\hline C.1. 1-20 & (CT)18 & $\begin{array}{l}\text { F: CGCGCGTGAGGACCCTATA } \\
\text { R: AGCAATTGATTGAGGCGGTTCT }\end{array}$ & 55 \\
\hline C.1. 2-23 & (CT)15 & $\begin{array}{l}\text { F: GAGGCGGAGGAGTTGAGAG } \\
\text { R: ACAAAACAACGAAACCCATAGC }\end{array}$ & 55 \\
\hline WGA3H & (TC)4 & $\begin{array}{l}\text { F: CGATAATCGACTTTCTCGAC } \\
\text { R: GCTTCTTAGCATCATCAACC }\end{array}$ & 55 \\
\hline
\end{tabular}

Les réactions d'amplification ont été effectuées dans un volume réactionnel de $15 \mu \mathrm{L}$ contenant 10 à $15 \mathrm{ng}$ de l'ADN génomique, de $2,25 \mathrm{mM}$ de $\mathrm{MgCl}_{2}$ (MP Biomedical, France), de $2 \mathrm{mM}$ de dNTPs (MP Biomedical, France), de 0,25 $\mu \mathrm{M}$ de l'amorce $\mathrm{R}$ (Eurogentec, France), de $10 \mu \mathrm{M}$ de l'amorce FM13 (Eurogentec, France), de $10 \mu \mathrm{M}$ de M13/700 ou 800 (Dye) (Eurogentec, France), de 0,5U de la Taq polymérase (MP Biomedical, France) et de 1X de PCR Buffer (MP Biomedical, France). Les amplifications ont été réalisées dans un thermocycler (T-Gradient thermoblock) programmé pour un cycle de $94^{\circ} \mathrm{C}$ à cinq minutes suivi de 40 cycles à $94^{\circ} \mathrm{C}$ pour 30 secondes, une 
minute de température d'hybridation qui a varié de 52 à $55^{\circ} \mathrm{C}$, une minute également pour l'élongation à $72^{\circ} \mathrm{C}$ et huit minutes pour l'élongation finale. Un contrôle sur gel d'agarose à $2 \%$ des produits de PCR a été réalisé afin de vérifier la qualité de l'amplification. La plaque a été mise à l'abri de la lumière à l'aide d'une feuille aluminium pour éviter la dégradation du fluorophore.

\section{Migration à l'aide d'un séquenceur à gel d'acrylamide}

Un gel polyacrylamide à $6,5 \%$ avec un peigne de 96 dents a été préparé et incorporé au séquenceur Li-Cor (NEN® model 4300 DNA Analyzer) après deux heures de polymérisation. C'est un séquenceur automatique à gel d'acrylamide qui permet de détecter, avec une bonne résolution, des fragments d'ADN de 50 à $700 \mathrm{pb}$ amplifiés soit avec le dye IR700 ou le dye IR800. Cinq microlitres de tampon renfermant $95 \%$ de formamide, $20 \mathrm{mM}$ EDTA et $0,05 \%$ de xylène cyanol ont été ensuite ajoutés aux produits de la PCR. Les échantillons ont d'abord été dénaturés sur une plaque chauffante $94^{\circ} \mathrm{C}$ pendant trois minutes, distribués sur un Rack aspirés par capillarité sur un peigne membrane de 100 dents et déposés sur le gel d'acrylamide préalablement monté sur le séquenceur. Les allèles ont été ensuite séparés par électrophorèse verticale haut voltage $\left(1500 \mathrm{~V}, 35 \mathrm{~mA}, 31 \mathrm{~W}\right.$ à $50^{\circ} \mathrm{C}$ pendant une heure 30 minutes) en fonction de leur taille. La détection des allèles se fait grâce aux deux lasers du séquenceur Li-Cor et la visualisation de la migration a été possible grâce à un ordinateur connecté au séquenceur. Les images des gels stockées sur le séquenceur ont été exportées sur l'ordinateur pour la lecture des profils. L'analyse des profils de migration a été effectuée grâce au logiciel $\mathrm{SAGA}^{\mathrm{GT}}$. Il s'agit d'un programme Li-Cor qui est un outil développé pour la lecture des profils de microsatellites migrés sur le séquenceur Li-Cor chez les organismes diploïdes.

\section{Analyse statistique des données}

Les différentes analyses utilisées dans cette étude ont pour objectif de déterminer dans un premier temps le déterminisme génétique et ensuite le nombre de QTL impliqués dans la tolérance de $L$. siceraria aux insectes.

Le déterminisme génétique a été étudié en utilisant la méthode de Bezawada et al. (2003) en appliquant le test khi-carré $\left(\chi^{2}\right)$. Le test de khi-carré $\left(\chi^{2}\right)$ pour le ratio phénotypique a été calculé en utilisant la formule suivante :

$$
\chi_{\text {calculé }}^{2}=\frac{(O-E)^{2}}{E}
$$

Où $\mathrm{O}$ est l'effectif observée et $\mathrm{E}$ l'effectif attendu.

Etant donné qu'il n'y a seulement que deux classes génotypiques pour les deux types de dégâts foliaires (dégâts foliaires élevés et dégâts foliaires insignifiants), le nombre de degré de liberté était égal à l'unité, de sorte que 
pour chaque test khi-carré, la différence a été considérée comme significative $(P<0,05)$ si sa valeur calculée était supérieure à 3,84 .

Le test était basé sur l'hypothèse nulle $\left(\mathrm{H}_{0}\right)$ d'égalité entre les proportions des deux catégories de feuilles (feuilles porteuses de dégâts et feuilles non porteuses de dégâts) et la distribution $3: 1$ ou encore $75 \%: 25$ $\%$. Admettre cette distribution revient à supposer que le caractère est gouverné par un gène à deux allèles, avec dominance complète. La tolérance des génotypes aux attaques d'insectes a été considérée comme dominante avec 75 $\%$ et $25 \%$ pour la récessivité.

Le rejet de l'hypothèse nulle intervenait quand le khi-carré calculé a été plus grand que le khi-carré théorique. Dans ce cas, l'hypothèse d'un couple d'allèles avec dominance complète a été être rejetée.

La seconde analyse pour identifier les marqueurs moléculaires a été effectuée sur les individus de la F2. La comparaison des moyennes du niveau des dommages foliaires des individus de la F2 a été effectuée entre les différentes classes génotypiques de chaque marqueur. Lorsque deux classes génotypiques pour chaque marqueur ont été observées, alors le test $t$ de Student a été utilisé pour la comparaison des moyennes. L'analyse de variance à un facteur (ANOVA1) a été effectuée pour trois classes génotypiques. S'il a existé une différence significative entre les différentes moyennes pour un marqueur donné, alors un QTL intervenant dans la tolérance des dommages des insectes a existé à proximité de ce marqueur. Dans le cas contraire, l'analyse effectuée n'a pas permis de mettre en évidence un QTL associé au marqueur. Tous les tests statistiques ont été réalisés à l'aide du logiciel SPSS 16.0 (SPSS, 2007).

\section{Résultats}

\section{Intensité des dégâts foliaires des individus parentaux et de la F2}

L'évaluation des dégâts foliaires dus aux insectes ravageurs des deux populations parentales et de la population F2 a été menée (Tableau 2). Le degré de sévérité des 150 individus a varié de 1 à 5 . Les individus parentaux des accessions NI431 et de NI227 ont été caractérisés respectivement par des dommages foliaires très faibles et des dommages foliaires élevés. Parmi les 100 individus de la F2, 72 ont été caractérisés par des dégâts très faibles tandis que l'intensité des dommages a été élevée et très élevée chez les 28 autres individus. 
Tableau 2. Récapitulatif des classes de sévérité des symptômes des accessions de Lagenaria siceraria aux insectes ravageurs

\begin{tabular}{clcccccc}
\hline & \multicolumn{6}{c}{ Classes phénotypiques } & \\
\cline { 2 - 7 } Populations & $\begin{array}{c}\mathbf{1} \\
\text { (dégâts très } \\
\text { faibles) }\end{array}$ & $\begin{array}{c}\mathbf{2} \\
\text { (dégâts } \\
\text { faibles) }\end{array}$ & $\begin{array}{c}\mathbf{3} \\
\text { (Intermédiaire }\end{array}$ & $\begin{array}{c}\mathbf{4} \\
\text { (dégâts } \\
\text { élevés) }\end{array}$ & $\begin{array}{c}\mathbf{5} \\
\text { (dégâts } \\
\text { très élevés) }\end{array}$ & Total \\
\hline \multirow{3}{*}{ Effectif } & $\begin{array}{l}\text { NI43 } \\
\mathbf{1}\end{array}$ & 25 & 0 & 0 & 0 & 0 & 25 \\
\cline { 2 - 7 } & $\mathbf{N I 2 2}$ & 0 & 0 & 0 & 13 & 12 & 25 \\
& $\mathbf{7}$ & 72 & 0 & 0 & 25 & 3 & 100 \\
\hline
\end{tabular}

1: dégâts très faibles; 2: dégâts faibles; 3: dégâts intermédiaires; 4: dégâts élevés; 5: dégâts très élevés

Déterminisme génétique de la tolérance des dégâts des insectes ravageurs

La ségrégation observée était de $72: 28$ et le khi-carré relatif à la ségrégation attendue ( $3: 1$ dégâts foliaires faible : dégâts foliaires élevés) était de 0,48 . Comme la valeur du khi-carré observé $\left(\chi^{2}\right.$ calculé $\left.=0.48\right)$ a été inférieure à celle du khi-carré théorique $\left(\chi^{2}\right.$ théorique $\left.=3,84\right)$, l'hypothèse nulle $\mathrm{H}_{0}$ a été acceptée. Autrement dit, il est probable que le caractère tolérance de $L$. siceraria aux attaques d'insectes ravageurs soit contrôlé par un couple d'allèles, avec dominance complète.

\section{Polymorphisme des marqueurs microsatellites utilisés}

Six marqueurs SSR ont été utilisés pour le génotypage de150 individus. Quatre d'entre eux ont été polymorphes c'est-à-dire les marqueurs EST00680, C1.1.20, C1.2.23 et UFR. Les deux autres marqueurs EST00691 et WGA3A ont été monomorphes. Ils n'ont pas été considérés dans l'analyse statistique parce qu'ils n'ont pas été informatifs. Au total neuf allèles ont été identifiés dans les 3 populations : population parentale NI431, population parentale NI227 et population en ségrégation F2. Le nombre d'allèles par locus a varié de 2 à 3 . Un effectif de 2 allèles a été observé au niveau des loci EST00680, C1.2.23 et UFR. Trois allèles ont été observés chez le locus C1.1.20 (Tableau 3). 
Tableau 3. Polymorphisme des marqueurs microsatellites utilisés dans l'étude des populations

parentales et F2 de Lagenaria siceraria

\begin{tabular}{lcc}
\hline Marqueurs moléculaires & Nombre d'allèles & Identité des allèles \\
\hline EST00680 & 2 & 222 \\
\hline & & 224 \\
\hline CI.1.20 & 3 \\
\hline & 2 & 147 \\
\hline CI.2.23 & 165 \\
\hline UFR & 168 \\
\hline & 2 & 194 \\
\hline
\end{tabular}

\section{Recherche de QTLs associés à la tolérance des dégâts des insectes}

La comparaison des moyennes du niveau des dommages foliaires des individus de la F2 a été effectuée entre les différentes classes génotypiques de chaque marqueur moléculaire (Tableau 4 et 5).

Le test $t$ de Student réalisé pour le marqueur EST00680 n'a pas montré de différence significative $(t=1,833 ; P=0,772)$ entre les moyennes de la sévérité des dégâts des insectes des deux génotypes $222 / 222$ et $224 / 224$. Il n'a pas existé de QTL à proximité de ce marqueur.

L'analyse de variance réalisé pour le marqueur $\mathrm{C} 1.1 .20$ a révélé qu'aucune différence significative $(F=0,776 ; P=0,512)$ n'a été observée entre les moyennes de la sévérité des dégâts des insectes des quatre génotypes $147 / 147,147 / 165,165 / 165$ et 168/168. Il n'a donc pas eu également de QTL à proximité de ce marqueur.

Le test $t$ de Student réalisé pour le marqueur C1.2.23 a montré une différence significative $(t=3.766 ; P=0.001)$ entre les moyennes de la sévérité des dégâts des insectes des deux génotypes 194/194 et 224/224. Il y’a donc un QTL identifié à proximité de ce marqueur.

Quant au marqueur UFR, le test $t$ de Student $\mathrm{n}$ 'a pas indiqué de différence significative entre les moyennes de la sévérité des dégâts causés par les insectes des deux génotypes 232/248 et 248/248. Aucun QTL n'a été identifié à proximité de ce marqueur. 
Tableau 4. Recherche de QTL par comparaison à partir du test $t$ des génotypes des différents marqueurs

\begin{tabular}{|c|c|c|c|c|c|}
\hline Marqueurs moléculaires & $\begin{array}{c}\text { Classes } \\
\text { Génotypiques }\end{array}$ & Effectifs & $\begin{array}{c}\text { Valeur } \\
\text { moyenne du } \\
\text { niveau de } \\
\text { dommage }\end{array}$ & $t$ & $P$ \\
\hline \multirow[b]{2}{*}{ EST00680 } & $222 / 222$ & 59 & $2,100 \pm 1,620$ & \multirow[t]{2}{*}{1,833} & \multirow[t]{2}{*}{0,772} \\
\hline & $224 / 224$ & 6 & $1,000 \pm 0,000$ & & \\
\hline \multirow[b]{2}{*}{ C1.2.23 } & $194 / 194$ & 54 & $0,91 \pm 1,581$ & \multirow[t]{2}{*}{3,766} & \multirow[t]{2}{*}{$<0,001$} \\
\hline & $224 / 224$ & 61 & $2,11 \pm 1,827$ & & \\
\hline \multirow[t]{2}{*}{ UFR } & $248 / 248$ & 101 & $2,440 \pm 1,670$ & 1,860 & 0,650 \\
\hline & $232 / 248$ & 15 & $1,600 \pm 1,242$ & & \\
\hline
\end{tabular}

Tableau 5. Recherche de QTL par comparaison à partir de l'ANOVA des génotypes du marqueur C1.1.20

\begin{tabular}{|c|c|c|c|c|c|}
\hline Marqueur moléculaire & $\begin{array}{c}\text { Classe } \\
\text { Génotypique }\end{array}$ & Effectifs & $\begin{array}{c}\text { Valeur } \\
\text { moyenne du } \\
\text { niveau de } \\
\text { dommage }\end{array}$ & $F$ & $\boldsymbol{P}$ \\
\hline \multirow{3}{*}{ C1.1.20 } & $147 / 147$ & 3 & $1,000 \pm 0,000$ & \multirow[t]{3}{*}{0,776} & \multirow[t]{3}{*}{0,512} \\
\hline & $147 / 165$ & 5 & $1,600 \pm 1,342$ & & \\
\hline & $165 / 165$ & 46 & $2,130 \pm 1,586$ & & \\
\hline
\end{tabular}

\section{Discussion}

Le criblage des différents génotypes de Lagenaria siceraria aux insectes defoliateurs a montré que les individus parentaux de NI431 et NI227 ont présenté des caractères bien contrastés au niveau de l'intensité des dégâts observés sur les feuilles. Ceci est manifesté par la diversité génétique observée chez L. siceraria comme l'avaient observé Koffi et al. (2009). Cette diversité génétique a permis d'observer une plus grande variabilité phénotypique dans la génération F2.

La réaction des individus de la F2 (issus du croisement entre l'accession NI431 et l'accession NI227) aux insectes a montré une ségrégation de $3: 1$ indiquant que l'aptitude de la plante à se défendre contre les attaques des insectes ravageurs est contrôlée par un seul gène, l'allèle conférant cette tolérance étant dominant. Ainsi, dans l'expression de la tolérance ou de la sensibilité face aux attaques des insectes sur L. siceraria, le phénotype tolérant [S] a dominé le phénotype sensible [s]. Ce résultat a suggéré que dans la population $\mathrm{F} 2$, un seul gène avec deux allèles : $\mathrm{S}$ (provenant de 1'accession NI431), et s (issu de NI227) a été identifié dans le contrôle de la tolérance des dégâts des insectes. La mise en évidence d'un seul gène dans la lutte contre les dégâts des bio-agresseurs a été observée chez le tabac (Zhang et al., 2008). Dans leurs travaux sur la tolérance du niébé à la plante parasite Striga, des chercheurs ont émis l'hypothèse qu'un même gène pourrait contrôler la 
résistance à différentes races de ce parasite (Touré et al., 1997). Par conséquent, vu que plusieurs insectes ravageurs ont occasionné des dégâts sur L. siceraria, le couple de gènes $\mathrm{S} / \mathrm{s}$ identifié dans la présente étude contrôlerait à lui seul la tolérance contre les attaques de ces ravageurs. Selon Kim (1998), la recherche d'une résistance horizontale (résistance polygénique) serait plus durable que la recherche d'une résistance verticale (monogénique), puisque cette dernière est facilement contournée et déjouée par le parasite. Dans le cas de la présente étude, le déterminisme génétique de type monogénique induisant une résistance verticale mis en évidence dans la tolérance des attaques des insectes ravageurs pourrait être un atout dans l'amélioration de $L$. siceraria puisque selon Parker (1991) il est facile et rapide de transférer ce type de résistance à des variétés aux propriétés agronomiques intéressantes. Le caractère tolérance de $L$. siceraria aux attaques d'insectes ravageurs a obéit aux règles mendéliennes classiques. Les allèles $\mathrm{S}$ et $\mathrm{s}$ seraient donc localisés sur les chromosomes (L'Héritier, 1975). L'hérédité chromosomique de ce caractère mise ici en évidence a démontré que les allèles $S$ et $\mathrm{s}$ ne sont pas contrôlés par des organites cellulaires tels les mitochondries et les chloroplastes. Cela oriente davantage les sélectionneurs en privilégiant dorénavant la transmission chromosomique plutôt que maternelle dans le schéma d'amélioration de $L$. siceraria contre des attaques des insectes ravageurs.

La détection de QTL de tolérance des dégâts causés par les insectes ravageurs est un préambule pour déterminer les gènes impliqués et pour mieux comprendre les voies biochimiques dans les mécanismes de défense de $L$. siceraria aux insectes. Les marqueurs moléculaires les plus couramment utilisés sont les microsatellites. Ces marqueurs sont hautement polymorphes par la variation du nombre de répétition et de ce fait ont été utilisés dans de nombreuses études d'identification des QTL (Selvaraj et al., 2011 ; Darvishzadeh, 2012). L'utilisation des marqueurs moléculaires SSR appliqués aux plantes de L. siceraria soumises aux insectes a permis de déterminer un seul QTL nommé qTDco. Le nombre de QTL identifié dans cette étude est très faible. Une détection robuste de QTL nécessite une saturation maximale et une distribution des marqueurs sur la carte génétique (Julio, 2005). Chez la cucurbite Citrullus lanatus, Hashizume et al. (2003) ont utilisé 554 marqueurs moléculaires pour identifier 5 QTLs liés aux paramètres agronomiques. Le faible nombre de QTL obtenu dans le cas de la présente étude pourrait être attribué au faible nombre de marqueurs SSR utilisés. Les marqueurs utilisés ne couvrent pas l'ensemble du génome de L. siceraria. L'utilisation d'un nombre accru de marqueurs moléculaires dans la détection de QTL de la tolérance des dégâts de $L$. siceraria face aux insectes ravageurs serait nécessaire dans le but d'accroitre le taux de saturation du génome de $L$. siceraria. Néanmoins, le marqueur C1.2.23 associé à ce QTL serait un outil 
indispensable pour de nombreuses perspectives en matière de sélection assistée par marqueur. Ce marqueur pourrait être exploité pour suivre des générations successives en vue de sélectionner les plantes porteuses des gènes de la tolérance.

\section{Conclusion}

L'objectif principal de cette étude a été la recherche des marqueurs moléculaires de type SSR associés au QTL de la tolérance contre les attaques d'insectes ravageurs. L'hérédité de l'expression de la tolérance ou la sensibilité vis-à-vis des attaques des insectes chez $L$. siceraria a été chromosomique et sous l'influence d'un couple d'allèle $S /$ s ou le phénotype tolérant [S] a dominé le sensible [s]. Aussi, un seul QTL nommé qTDco a t-il été révélé à partir du marqueur moléculaire $\mathrm{C} 1.2 .23$. Ce marqueur a donc été associé aux gènes de la tolérance de Lagenaria siceraria aux dégâts des insectes ravageurs. Ce marqueur pourrait être utilisé pour procéder à une sélection précoce et rapide des plantes de $L$. siceraria ayant le gène de la tolérance aux insectes ravageurs. Il apparait indispensable d'initier une autre étude visant à accroitre le taux de saturation du génome de $L$. siceraria par l'utilisation de nombreux marqueurs moléculaires.

\section{References:}

1. Achigan Dako GE, Fanou N, Kouke A, Avohou H, Vodouhe RS \& Ahanchede A (2006). Evaluation agronomique de trois espèces de Egusi (Cucurbitaceae) utilisées dans l'alimentation au Bénin et élaboration d'un modèle de prédiction du rendement. Biotechnologie Agronomie Société Environnement 10 (2): 121-129.

2. Akpavi S, Kanda M, Odah K, Akpakpah KE, Kossi-Titrikou, Boutare I \& Akpagora k (2013).Valeur socio-culturelle des plantes alimentaires: un facteur de préservation. European Scientific Journa 19 (32): 383-395

3. Anzara GKGR, Koffi KK, Coulibaly SS, Fouah BI ND, Baudoin J-P, Campa C \& Zoro BI IA (2015). Influence of herbivorous insects on the production of Lagenaria siceraria (Molina) Standley (Cucurbitaceae).African Journal of Plant Science 9 (11): 450-456

4. Baudoin JP (2001). Contribution des ressources phytogénétiques à la sélection variétale de légumineuses alimentaires tropicales. Biotechnology, Agronomy, Society and Environnement 5 (4): 221-230.

5. Bezawada C, Saha S, Johnie N, Roy G and McCarty J (2003).SSR Marker(s) associated with root knot nematode resistance gene(s) in cotton. The Journal of Cotton Science 7:179-184

6. Darvishzadeh R (2012). Association of SSR markers with partial resistance to Sclerotinia sclerotiorum isolates in sunflower 
(Helianthus annuus L.). Australian Journal of Crop Science6 (2): 276282.

7. Julio (2005). Développement d'une carte génétique de Nicotiana tabacum et identification de QTLs liés à des caractères agronomiques et à la composition de la fumée. Toulouse (France): Institut National Polytechnique; 114 pages.

8. Kim SK (1998). Horizontal resistance: misunderstandings, approach and importance. In: Kim SK, Robinson RA, Atkinson K, Adetimirin VO, Thé $C$ \&Sallé $G(E d s)$ Combating parasitic weeds through horizontal resistance. Seoul(Repubique de Coré): International Agriculture Research Institute, pp 53-56.

9. Koffi KK, Anzara GK, Malice M, Djè Y, Bertin P, Baudoin JP \& Zoro Bi IA (2009). Morphological and allozyme variation in a collection of Lagenaria siceraria (Molina) Standl. from Côte d'Ivoire. Biotechnology Agronomy Society and Environment 13 (2): 257-270.

10. Kouassi NJ \& Zoro BIA (2009). Effect of sowing density and seedbed type on yield and yield components in bambara groundnut (Vigna Subterranea) in woodland savannas of Côte d'Ivoire. Experimental Agriculture 46 (1): 99-110.

11. L’Héritier P (1975). Génétique. Paris (France): Masson et Cie; 314 pages.

12. Loukou AL, Gnakri D, Djè Y, Kippré AV, Malice M, Baudoin JP \& Zoro Bi IA (2007). Macronutrient composition of three cucurbit species cultivated for seed consumption in Côte d'Ivoire. African Journal of Biotechnology 6 (5): 529-533.

13. Murray MG \& Thompson WF (1980). Rapid isolation of high molecular weight plant DNA. Nucleic Acids Research 8 (19): 43214326.

14. Najimi B, Boukhatem N, El Jaafari S, Jlibène M, Paul R \& Jacquemin JM (2002). Amplified fragment length polymorphism (AFLP) analysis of markers associated with $\mathrm{H} 5$ and $\mathrm{H} 22$ Hessian fly resistance genes in bread.Biotechnologie, Agronomie, Société et Environnement 6 (2): 7985.

15. Ndabalishye I (1995). Agriculture vivrière ouest africaine à travers le cas de la Côte d'Ivoire. IDESSA: Bouaké (Côte d'Ivoire); 255 pages.

16. Parker C (1991). Protection of crop against parasitic weeds. Crop Protection 10:6-10

17. Pitan OOR \& Ekoja EE (2011). Yield response of okra, Abelmoschus esculentus (L.) Moench to leaf damage by the flea beetle, Podagrica uniforma Jacoby (Coleoptera: Chrysomelidae). Crop Protection 30: 1346-1350. 
18. Rasul GM, Hiramatsu M \& Okubo H (2007). Genetic relatedness (diversity) and cultivar identification by randomly amplified polymorphic DNA (RAPD) markers in teasle gourd (Momordica dioica Roxb.). Scientia Horticulturae 111: 271-279.

19. Rouxel T, Penaud A, Pinochet X, Brun H, Gout L, Delourme R, Schmit J \& Balesdent MH (2003).A 10-year survey of populations of Leptosphaeria maculans in France indicates a rapid adaptation towards the Rlm1resistance gene of oilseed rape. European Journal of Plant Pathology109: 871-881.

20. Selvaraj IC, Nagarajan P, Thiyagarajan K, Bharathi M \& Rabindran R (2011). Identification of Microsatellite (SSR) and RAPD Markers Linked to Rice Blast Disease Resistance gene in Rice (Oryza sativa L.). African Journal of Biotechnology10 (17): 3301-3321.

21. SPSS (2007). SPSS 16.0 for windows version 16.0. Chicago, USA: SPSS Inc., 233 south wacker drive, 11th Floor. Chicago, USA.

22. Touré M, Olivier A, Ntare BR, Lane JA \& St-Pierre CA (1997). Meritance of resistance to Striga gesnerioides biotypes from Mali and Niger in cowpea (Vigna mguiculata (L.) Walp.). Euphytica94: 273278.

23. Vayssieres JF, Delvare G, Maldès JM \& Aberlenc HP (2000). Inventaire preliminaire des arthropodes ravageurs et auxiliaires des cultures maraicheres sur l'ile de la reunion. Insect Science and its Application 21 (1): 1-22.

24. Zhang HY, Yang YM, LI FS, He CS \& Liu XZ (2008). Screening and characterization a RAPD marker of tobacco brown-spot resistant gene. African Journal of Biotechnology7 (15): 2559-2561.

25. Zoro Bi IA, Djé Y, Koffi KK, Djé Y, Malice M \& Baudoin JP (2006). Indigenous cucurbits of Côte d'Ivoire: a review of their genetic resources. Sciences et Nature3 (1): 1-9.

26. Zoro Bi IA, Koffi KK \& Djé Y (2003). Caracterisation botanique et agronomique de trois espèces de curcubites consommées en sauce en Afrique de l'ouest:Citrullus sp., Cucumeropsis mannii Naudin et Lagenaria siceraria (Molina.) Standl. Biotechnology, Agronomy, Society and Environnement7: 189-199. 